\title{
Modeling of temperature sensor built on thin silicon on insulator using advanced carrier-mobility model
}

\author{
Z. H. Wu and P. T. Lai ${ }^{\text {a) }}$ \\ Department of Electrical and Electronic Engineering, The University of Hong Kong, \\ Hong Kong Special Administrative Region of China
}

(Received 9 February 2004Received 27 May 2004)

Based on the minority-carrier exclusion theory, an analytical model is developed to explain the operating principle of temperature sensors fabricated on thin silicon film. The model also takes into account the effects of temperature and carrier concentrations on carrier mobilities. Device simulation is used to verify the proposed model in terms of the carrier-concentration distribution in the minority-exclusion region, exclusion length, and the temperature dependence of the sensor resistance. Results show that the model is applicable for a sensor operating over a wide temperature range, provided that its bias current is not too high. (C) 2004 American Institute of Physics.

[DOI: 10.1063/1.1774266]

\section{INTRODUCTION}

At present, there is a growing demand for electronic devices that can function over a wide temperature range, for example, in the high-temperature compartments of automobiles and aircrafts. High-temperature sensors based on wideband-gap semiconductors, such as SiC, diamond, ${ }^{1,2}$ and fibers, ${ }^{3}$ have been investigated in attempts to produce electronic devices that operate reliably even at high temperatures. However, it would still be a long time before wideband gap semiconductor sensors can become cost-effective. Also, there are numerous problems for fiber sensors to be integrated into a single-chip system. Therefore, the development of high-temperature sensors based on the prevalent silicon and related technologies is still attracting a lot of attention.

Silicon devices are normally only suitable for operating below temperatures of $250^{\circ} \mathrm{C}$ due to the excessive thermal generation of charge carriers at high temperatures. ${ }^{4}$ However, by introducing the spreading-resistance structure, the maximum operating temperature $\left(T_{\max }\right)$ of silicon temperature sensors can be significantly increased. The spreadingresistance temperature (SRT) sensor was introduced ${ }^{5}$ in 1982 and commercialized ${ }^{6}$ in 1983 . This SRT sensor has a vertical structure as shown in Fig. 1(a), and can operate at temperatures from $-65^{\circ} \mathrm{C}$ to $300^{\circ} \mathrm{C}$. Later on, Hout and Middelhoek ${ }^{7}$ raised the $T_{\max }$ of their SRT sensors to $400^{\circ} \mathrm{C}$. Moreover, they developed a one-dimensional (1D) minoritycarrier exclusion model to explain the principle of spreading resistance and the characteristics of the vertical temperature sensors. $^{7,8}$

Recently, the lateral temperature sensor in Fig. 1(b) fabricated by thin-film silicon-on-insulator (SOI) processing ${ }^{9-11}$ was studied, and its $T_{\max }$ ranges from $300^{\circ} \mathrm{C}$ to $450^{\circ} \mathrm{C}$. Besides possessing a high working temperature, the lateral temperature sensor is also suitable for integration into a microchip due to its planar structure. The lateral temperature

\footnotetext{
a) Author to whom correspondence should be addressed; electronic mail: laip@eee.hku.hk
}

sensor presented here has a low-doping $n$ region with two electrodes forming ohmic contacts to the $n^{+}$regions at both ends. When a current flows through the two electrodes, the minority-carrier exclusion effect will occur due to the presence of the high-low junction. Although device simulation can be used to estimate the sensor's resistance vs temperature characteristics, a simple theoretical model is still needed to facilitate the design of the sensor. This work presents a

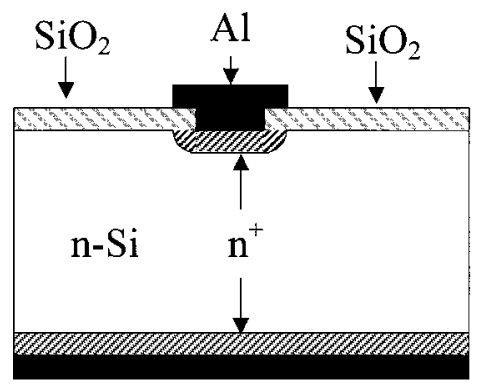

(a)

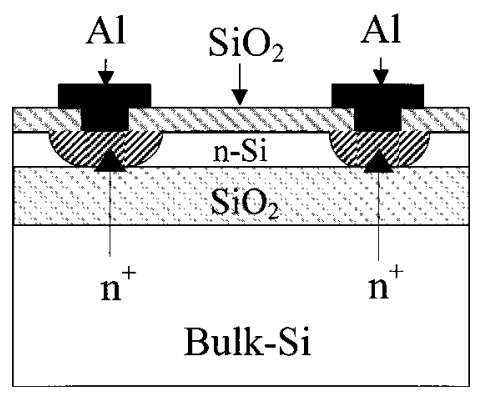

(b)

FIG. 1. Cross-sectional view of (a) vertical SRT sensor on bulk Si and (b) lateral temperature sensor on SOI. The vertical sensor has a small circular $n^{+}$ region at the top and a large $n^{+}$region at the bottom, forming a spreading resistor for increasing the maximum working temperature. Whereas for the lateral sensor, the two rectangular $n^{+}$regions are at the top of the SOI forming two high-low junctions. Since the silicon film is thin, high-density current flow from one electrode to another can produce strong minoritycarrier exclusion near one junction, and hence raise the maximum working temperature. 


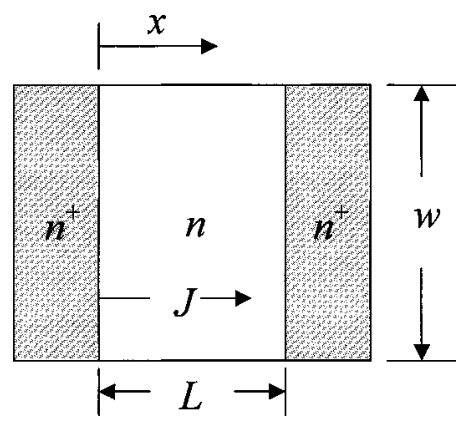

FIG. 2. Top view of the lateral temperature sensor on SOI. The $J$ is the current density applied to the sensor, $w$ is the width of the sensor, $L$ is the length of the sensor ( $n$ region), and $x$ is the distance from the $n^{+} n$ junction. In this work, $w$ is set to be $100 \mu \mathrm{m}$ whereas $L$ is set to be $10 \mu \mathrm{m}$.

physical model for the SOI temperature sensor, taking into account the minority-carrier exclusion effect, together with the temperature and carrier-concentration effects on carrier mobilities.

\section{THEORY}

The top view of the temperature sensor is shown in Fig. 2 , where $w$ is the width of the sensor and $L$ is the length of the $n$ region, with $x$ as the distance from the $n^{+} n$ junction. Since the electron concentration $n(x)$ of the $n^{+}$region $(x$ $\leqslant 0)$ is much higher than that of the $n$ region $(0 \leqslant x \leqslant L)$, the hole concentration $p(x)$ in the $n^{+}$region is much lower than that in the $n$ region. When an electric field is applied along the positive $x$ direction, minority carriers (holes) would flow along the $x$ direction whereas majority carriers (electrons) would move in the opposite direction. Near the $n^{+} n$ high-low junction $(x=0)$, holes in the $n$ region are extracted by the electric field at a faster rate than those supplied by the $n^{+}$ region due to the difference in hole concentration. Therefore, the hole concentration in the $n$ region near the junction is reduced. If the electric field is strong enough, this hole concentration near the junction will be lowered to nearly zero, even at high temperatures. Then, the electron concentration can be restored to the original doping level due to the condition of space-charge neutrality. This is the essence of the minority-carrier exclusion in the $n$ region next to the $n^{+} n$ junction, where thermally generated carriers are effectively removed. Since the extrinsic carriers dominate the electrical conduction, the resistance of the sensor remains the same as that before thermal generation due to high temperatures. This also explains why the $T_{\max }$ of the sensor can be raised to higher values. Since the minority-carrier concentration does not really go to zero when exclusion occurs, a parameter $\lambda$ is used to define the extent of exclusion. The exclusion regime is defined to be in effect when the minority-carrier concentration is reduced to $\lambda N_{\mathrm{D}}$, where $N_{\mathrm{D}}$ is the doping level of the $n$ region.

The well-known carrier transport equations based on the drift-diffusion model are

$$
J_{\mathrm{n}}=q \mu_{\mathrm{n}} n E+k T \mu_{\mathrm{n}} \nabla n,
$$

$$
J_{\mathrm{p}}=q \mu_{\mathrm{p}} p E-k T \mu_{\mathrm{p}} \nabla p
$$

where $E$ is the electric field, $k$ is the Boltzmann constant, $T$ is the operating temperature, $J_{\mathrm{n}}$ and $J_{\mathrm{p}}$ are the electron and hole current densities, and $\mu_{\mathrm{n}}$ and $\mu_{\mathrm{p}}$ are the electron and hole mobilities, respectively. Assuming space-charge neutrality is observed in the $n$ region, $n=N_{\mathrm{D}}+p$ and for uniform doping level, $\nabla n=\nabla p$.

With $J_{\mathrm{n}}=J-J_{\mathrm{p}}$ and mobility ratio $b \equiv \mu_{\mathrm{n}} / \mu_{\mathrm{p}}$, eliminating $E$ from Eqs. (1) and (2) gives

$$
J_{\mathrm{p}}=\frac{p J-k T \mu_{\mathrm{n}}(n+p) \nabla p}{b n+p} .
$$

Since the silicon film is very thin, the currents in the $y$ and $z$ directions can be assumed to be zero. In the carrier exclusion region, recombination can be ignored and the generation rate $G$ is constant. The continuity equation can be written as

$$
\frac{d J_{\mathrm{p}}}{d x}=q G
$$

Integrating both sides of Eq. (4) from 0 to $x$ results in

$$
J_{\mathrm{p}}=J_{\mathrm{p} 0}+q G x
$$

where $J_{\mathrm{p} 0}$ is the minority-carrier current density injected into the carrier-exclusion region at the $n^{+} n$ junction $(x=0)$ and is normally negligible. Substituting Eq. (5) into Eq. (3), using $n=p+N_{\mathrm{D}}$ (space-charge neutrality) and $J=I /\left(w t_{\mathrm{si}}\right)\left(t_{\mathrm{si}}\right.$ is the silicon-film thickness), and neglecting the diffusion term $(\nabla p)$ within the exclusion region, we can rewrite Eq. (3) as

$$
p=\frac{q G b N_{\mathrm{D}} x}{\frac{I}{w t_{\mathrm{si}}}-q(b+1) G x} .
$$

By setting the condition for the carrier exclusion region as $p \leqslant \lambda N_{\mathrm{D}}$ (where $\lambda$ is normally set to 0.1 ), the exclusion length at the $n^{+} n$ junction can be calculated as

$$
x_{\mathrm{ex}}=\frac{\lambda I}{w t_{\mathrm{si}}[q G b+\lambda q G(b+1)]} .
$$

Making use of Eq. (6) and $n=p+N_{\mathrm{D}}$, the resistivity of sensor is given by

$$
\rho=\frac{1}{n q \mu_{\mathrm{n}}+p q \mu_{\mathrm{p}}}=\frac{I-q G(b+1) w t_{\mathrm{si}} x}{q \mu_{\mathrm{n}} N_{D} I},
$$

where the material parameters are ${ }^{7,12}$

$$
\begin{aligned}
& G=\frac{1}{\tau N_{\mathrm{D}}+2 n_{\mathrm{i}}}, \\
& \mu_{\mathrm{n}}=55.24+\frac{1429.23\left(\frac{T}{300}\right)^{-2.3}-55.23}{1+\left(\frac{T}{300}\right)^{-3.8}\left(\frac{n+p}{1.072 \times 10^{17}}\right)^{0.73}},
\end{aligned}
$$




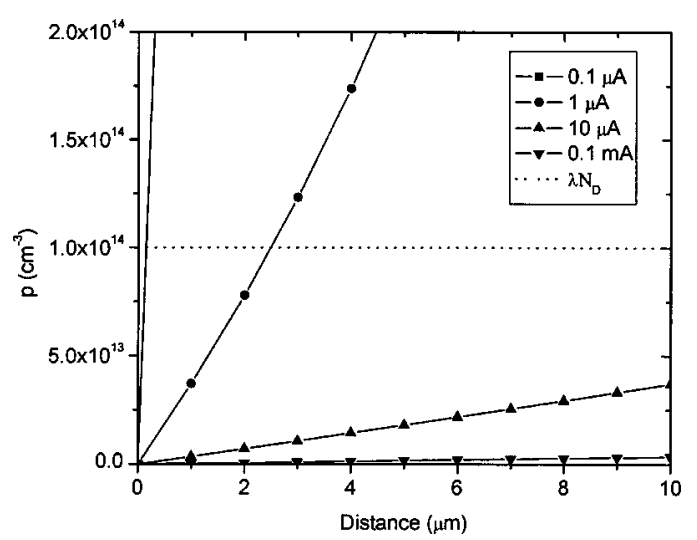

FIG. 3. Calculated minority-carrier concentration profiles across the temperature sensor at $300^{\circ} \mathrm{C}$ for bias currents of $0.1 \mu \mathrm{A}, 1 \mu \mathrm{A}, 10 \mu \mathrm{A}$, and $0.1 \mathrm{~mA}$. The dotted line $\left(\lambda N_{D}\right)$ defines the threshold level for minoritycarrier exclusion. In this work, the film doping level $\left(N_{D}\right)$ is $1 \times 10^{15} \mathrm{~cm}^{-3}$. The threshold level is defined to be one-tenth of the doping level $(\lambda=0.1)$. When the minority-carrier (hole) concentration falls below this level, the minority-carrier exclusion occurs.

$$
\mu_{\mathrm{p}}=49.7+\frac{479.37\left(\frac{T}{300}\right)^{-2.2}-49.7}{1+\left(\frac{T}{300}\right)^{-3.7}\left(\frac{n+p}{1.606 \times 10^{17}}\right)^{0.7}},
$$

and

$$
\begin{aligned}
& n_{\mathrm{i}}=\sqrt{N_{\mathrm{c}} N_{\mathrm{v}}} e^{-E_{\mathrm{g}} / 2 k T}, \\
& E_{\mathrm{g}}=E_{\mathrm{g}}(300 \mathrm{~K})+\alpha\left[\frac{300^{2}}{300+\alpha}-\frac{T^{2}}{T+\beta}\right], \\
& N_{\mathrm{c}}=N_{\mathrm{c}}(300 \mathrm{~K})\left(\frac{T}{300}\right)^{3 / 2}, \\
& N_{\mathrm{v}}=N_{\mathrm{v}}(300 \mathrm{~K})\left(\frac{T}{300}\right)^{3 / 2}
\end{aligned}
$$

The excess-carrier lifetime $\tau$ is taken to be $0.1 \mu$ s and the temperature coefficients for the energy band gap ( $\alpha$ and $\beta$ ) are $4.73 \times 10^{-4} \mathrm{eV} / \mathrm{K}$ and $636 \mathrm{~K}$, respectively. Finally, the resistance of the sensor can be calculated as

$$
R=\int_{0}^{L} \frac{\rho d x}{w t_{\mathrm{si}}}=\int_{0}^{L} \frac{I-q G(b+1) w t_{\mathrm{si}} x}{w t_{\mathrm{si}} q \mu_{\mathrm{n}} N_{\mathrm{D}} I} d x .
$$

\section{RESULTS AND DISCUSSIONS}

With $L$ of $10 \mu \mathrm{m}, w$ of $100 \mu \mathrm{m}$, and $t_{\mathrm{si}}$ of $0.1 \mu \mathrm{m}$, the distribution of minority-carrier concentration along the $x$ direction under different bias currents can be calculated from Eq. (6). The hole distributions in a SOI device at $300^{\circ} \mathrm{C}$ for different bias currents are plotted in Fig. 3. The dotted line defines the threshold level for minority-carrier exclusion, which occurs in the region where the hole concentration falls below this level. In this region, the electron concentration drops back to nearly the same as the original doping level because of space-charge neutrality. If the carrier exclusion

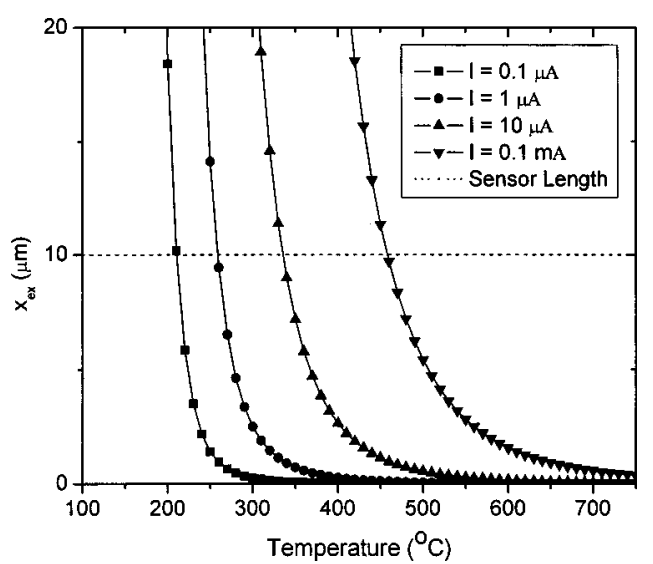

FIG. 4. Calculated carrier exclusion length for the temperature sensor with bias currents of $0.1 \mu \mathrm{A}, 1 \mu \mathrm{A}, 10 \mu \mathrm{A}$, and $0.1 \mathrm{~mA}$. The $N_{D}$ is 1 $\times 10^{15} \mathrm{~cm}^{-3}$. The dotted line shows the length of the sensor.

region is longer, the device needs a higher temperature to produce more electron and hole pairs in order to switch to intrinsic conduction, implying a higher $T_{\max }$. From Fig. 3 , it can easily be seen that when the current is larger, the exclusion length is larger and hence $T_{\max }$ is higher. When the bias current is at $0.1 \mu \mathrm{A}$, the hole concentration is higher than the threshold level within the entire $n$ region. This means that carrier exclusion does not occur if the bias current is too small, and $T_{\max }$ at this bias current should be far below $300^{\circ} \mathrm{C}$. When current is increased to $1 \mu \mathrm{A}$, carrier exclusion starts to occur near the $n^{+} n$ junction up to a distance of $2.5 \mu \mathrm{m}$. $T_{\max }$ will be a little higher than that for $0.1 \mu \mathrm{A}$, but still much lower than $300^{\circ} \mathrm{C}$. When the bias current is larger than $10 \mu \mathrm{A}$, carrier exclusion covers the entire $n$ region, and $T_{\max }$ at this high current bias will be higher than $300^{\circ} \mathrm{C}$.

Equation (7) evaluates the temperature dependence of the exclusion length under different bias currents as illustrated in Fig. 4. This is another way to display the relation between the carrier exclusion effect and $T_{\max }$. The temperature at which the carrier exclusion length (solid lines) intersects the length of the sensor (dotted line) represents the exact temperature that the carrier exclusion region covers the entire $n$ region of the sensor and is approximately equal to the $T_{\max }$ of the sensor at a given bias current. For instance, the intersection point of $0.1 \mu \mathrm{A}$ is $210^{\circ} \mathrm{C}$. When the temperature is below $210^{\circ} \mathrm{C}$, say $200^{\circ} \mathrm{C}$, the carrier exclusion length is larger than the length of the sensor. This means that the device needs more thermally generated electron-hole pairs to overwhelm the extrinsic carriers, and thus $T_{\max }$ should be higher than $200^{\circ} \mathrm{C}$. For temperature above $210^{\circ} \mathrm{C}$, say $220^{\circ} \mathrm{C}$, the carrier exclusion length is smaller than the length of the sensor, implying that part of the device has a lot of excess carriers. Therefore, $T_{\max }$ should be lower than $220^{\circ} \mathrm{C}$ at a current bias of $0.1 \mu \mathrm{A}$. Similar trends are also found for the other three bias currents, and $T_{\max }$ at different bias currents calculated by this method are as shown in Table I.

The integrand in Eq. (11) depends not only on the distance $x$ but also on the carrier mobilities $\mu_{\mathrm{n}}$ and $\mu_{\mathrm{p}}$, which are in turn functions of $p(x)$ in Eq. (6). Due to this complexity, the sensor's resistance has to be calculated by numerical 
TABLE I. $T_{\max }$ calculated by three methods for the temperature sensor at different bias currents

\begin{tabular}{lccc}
\hline \hline Bias currents & $\begin{array}{c}T_{\text {max }}\left({ }^{\circ} \mathrm{C}\right) \\
\text { from Fig. } 4\end{array}$ & $\begin{array}{c}T_{\max }\left({ }^{\circ} \mathrm{C}\right) \\
\text { from Eq. }(8)\end{array}$ & $\begin{array}{c}T_{\max }\left({ }^{\circ} \mathrm{C}\right) \\
\text { from PISCES }\end{array}$ \\
\hline $0.1 \mu \mathrm{A}$ & 210 & 210 & 230 \\
$1 \mu \mathrm{A}$ & 260 & 260 & 230 \\
$10 \mu \mathrm{A}$ & 350 & 360 & 330 \\
$0.1 \mathrm{~mA}$ & 460 & 510 & 430 \\
\hline \hline
\end{tabular}

method, which consumes much computer time and sometimes could have convergency problems. In the following description, different methods are proposed to simplify the calculation while maintaining acceptable accuracy.

\section{A. Case 1}

Since the intrinsic carrier concentrations are relatively low for temperatures not far beyond $T_{\max }$, the chargedependent term $n+p$ in the mobility formula can be approximated by $N_{\mathrm{D}}$, and thus $\mu_{\mathrm{n}}$ and $\mu_{\mathrm{p}}$ can be considered to be constant throughout the $n$ region (this will be later supported by Case 5). As a result, the resistance of the sensor in Eq. (11) can be analytically calculated as

$$
R=\frac{L}{q \mu_{\mathrm{n}} N_{\mathrm{D}} w t_{\mathrm{si}}}-\frac{G(b+1) L^{2}}{2 \mu_{\mathrm{n}} N_{\mathrm{D}} I} .
$$

The first term is the well-known formula for a simple diffused resistor, and it increases with temperature due to variation in $\mu_{\mathrm{n}}$. In the second term, $G$ accounts for the thermal generation of carriers, whereas $I$ produces the carrier exclusion effect. The combined effects on the characteristics of resistance vs temperature are as plotted in Fig. 5. The same characteristics simulated using the 2D device simulator PISCES are also included for comparison. Figure 5 shows that higher current induces stronger carrier exclusion effect, thus resulting in higher $T_{\max }$. The $T_{\max }$ calculated and simulated at different bias currents are also included in Table I for

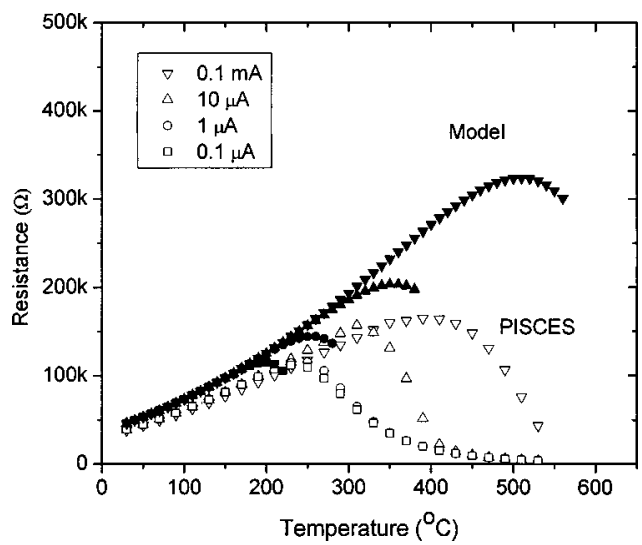

FIG. 5. Resistance-temperature curves for the temperature sensor with bias currents of $0.1 \mu \mathrm{A}, 1 \mu \mathrm{A}, 10 \mu \mathrm{A}$, and $0.1 \mathrm{~mA}$. The $N_{D}$ is $1 \times 10^{15} \mathrm{~cm}^{-3}$ Solid symbols show the calculated results of the simplified model with the charge-dependent term $n+p$ in the mobility formula approximated by $N_{D}$, and the mobilities $\mu_{n}$ and $\mu_{p}$ considered to be constant throughout the $n$ region. Hollow symbols are the results calculated by the $2 \mathrm{D}$ device simulator PISCES.

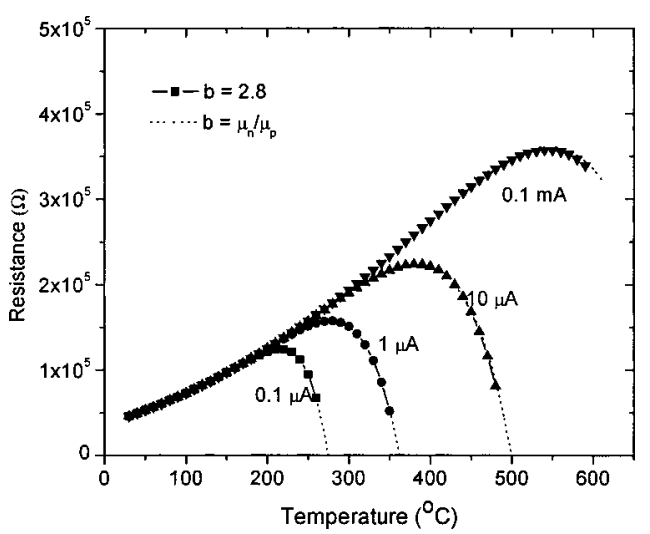

FIG. 6. Effects of temperature-sensitive carrier-mobility ratio $\left(b \equiv \mu_{n} / \mu_{p}\right)$ on sensor resistance at bias currents of $0.1 \mu \mathrm{A}, 1 \mu \mathrm{A}, 10 \mu \mathrm{A}$, and $0.1 \mathrm{~mA}$. The $N_{D}$ is $1 \times 10^{15} \mathrm{~cm}^{-3}$. Dotted lines show the results with the effect of temperature on $b$. Solid symbols show the results without the effect of temperature on $b$ ( $b$ is set at its room-temperature value of 2.8).

comparison. When the bias current is smaller than $0.1 \mathrm{~mA}$, there is little difference among the $T_{\max }$ calculated by the three methods. However, large discrepancies exist when the bias current is over $0.1 \mathrm{~mA}$. The reason is that when the bias current is large, the minority-carrier injection current density $J_{\mathrm{p} 0}$ in Eq. (5) can no longer be ignored. This carrier injection can increase the carrier concentrations in the $n$ region and thus lower its resistance as shown in Fig. 5.

\section{B. Case 2}

The carrier mobilities calculated by Eqs. (9) and (10) show that when the temperature increases from $30^{\circ} \mathrm{C}$ to $600^{\circ} \mathrm{C}$, their ratio $b$ only changes from 2.8 to 2.6. Therefore, $b$ can be further simplified as a constant and take on its room-temperature value of 2.8 . Since $\mu_{\mathrm{n}}$ and $\mu_{\mathrm{p}}$ are considered as constants throughout the $n$ region, Eq. (12) is still applicable for this case. The new characteristics of resistance vs temperature are as shown in Fig. 6, illustrating that the mobility ratio hardly affects the resistance.

\section{Case 3}

Owing to the approximations used in the model derivation, there exists a singularity $\left(x_{0}\right)$ in the hole-concentration distribution described by Eq. (6). When $x=x_{0} \equiv I /[q(b$ $\left.+1) G w t_{\mathrm{si}}\right], p$ will approach infinity. Since $p$ is negative for $x>x_{0}$, the integration range in Eq. (11) has to be adjusted from 0 to $x_{\text {up }}$, and Eq. (12) becomes

$$
R=\frac{x_{\mathrm{up}}}{q \mu_{\mathrm{n}} N_{\mathrm{D}} w t_{\mathrm{si}}}-\frac{G(b+1) x_{\mathrm{up}}^{2}}{2 \mu_{\mathrm{n}} N_{\mathrm{D}} I},
$$

where $x_{\text {up }} \equiv \min \left(L, x_{0}\right)$. As can be observed in Fig. 7, there is a little effect of $x_{0}$ on the resistance for temperatures below $T_{\max }$. This also confirms that the region from 0 to $x_{0}$ governs the resistance of the sensor when carrier exclusion effect occurs. For temperatures beyond $T_{\max }$, Eq. (13) gives a more realistic resistance-temperature characteristics than Eq. (12) by removing all the negative carrier concentrations. 


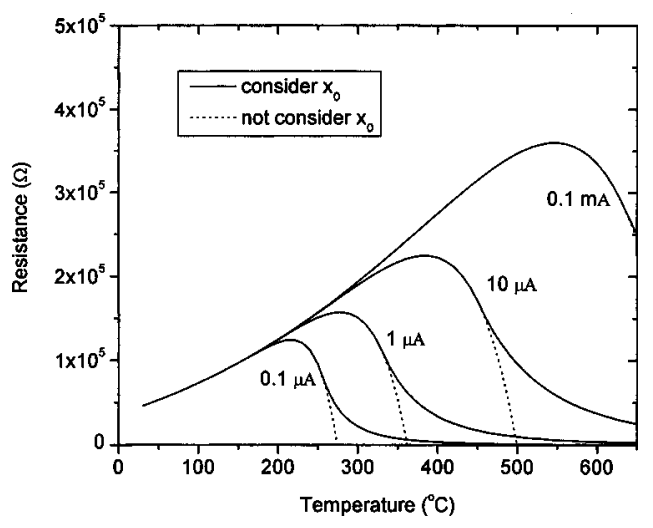

FIG. 7. Effects of carrier-concentration singularity on sensor resistance at bias currents of $0.1 \mu \mathrm{A}, 1 \mu \mathrm{A}, 10 \mu \mathrm{A}$, and $0.1 \mathrm{~mA}$. The $N_{D}$ is 1 $\times 10^{15} \mathrm{~cm}^{-3}$. Dotted lines are the results with negative values in the carrierconcentration profile inside the sensor, whereas solid lines show the results with negative concentrations removed.

\section{Case 4}

As mentioned in Case 3, $p$ in Eq. (6) rises rapidly to a very large value before becoming negative. But in reality, $p$ cannot be higher than its equilibrium value $p_{0}$, which is defined as ${ }^{4}$

$$
p_{0}=n_{\mathrm{i}}^{2} / n_{0}=\frac{n_{\mathrm{i}}^{2}}{\frac{1}{2}\left[N_{\mathrm{D}}+\sqrt{N_{\mathrm{D}}^{2}+4 n_{\mathrm{i}}^{2}}\right]},
$$

where $n_{0}$ is the equilibrium electron concentration. If $x_{\mathrm{p} 0}$ is defined as the location where $p$ in Eq. (6) increases to $p_{0}$, then

$$
x_{\mathrm{p} 0}=\frac{p_{0} I}{\left[q G b N_{\mathrm{d}}+q G(b+1) p_{0}\right] w t_{\mathrm{si}}} .
$$

For $x$ larger than $x_{\mathrm{p} 0}, p$ in Eq. (6) is larger than $p_{0}$ and so is set equal to $p_{0}$. The resistivity determined by Eq. (8) for this region becomes

$$
\rho_{0}=\frac{b}{q \mu_{\mathrm{n}} b N_{\mathrm{d}}+q \mu_{\mathrm{n}}(1+b) p_{0}} .
$$

With $x_{\max } \equiv \min \left(L, x_{\mathrm{p} 0}\right)$, Eq. (11) becomes

$$
\begin{aligned}
R= & \frac{1}{w t_{\mathrm{si}}}\left[\int_{0}^{x_{\max }} \rho(x) d x+\int_{x_{\max }}^{L} \rho_{0} d x\right]=\frac{x_{\max }}{q \mu_{\mathrm{n}} N_{\mathrm{d}} w t_{\mathrm{si}}} \\
& -\frac{G(b+1) x_{\max }^{2}}{2 \mu_{\mathrm{n}} N_{\mathrm{d}} I}+\frac{1}{w t_{\mathrm{si}}} \frac{b}{q \mu_{\mathrm{n}} b N_{\mathrm{d}}+q \mu_{\mathrm{n}}(1+b) p_{0}} \\
& \times\left(L-x_{\max }\right) .
\end{aligned}
$$

From Fig. 8, a big difference is found when the bias current is low (e.g., for $0.1 \mu \mathrm{A}, T_{\max }$ is $20^{\circ} \mathrm{C}$ higher when considering $\left.x_{\mathrm{p} 0}\right)$. For a lower bias current, since the carrier exclusion effect is weak, the hole concentration at high temperature given by Eq. (6) is much higher than $p_{0}$ for most of the integration range, given that $x_{\mathrm{p} 0}$ is much smaller than $L$. Hence, the results calculated by Eq. (12) are different from those calculated by Eq. (17) due to the significance of the last term in Eq. (17). For higher bias currents in the range of $1 \mu \mathrm{A}$ to $0.1 \mathrm{~mA}$, the two methods give similar $T_{\max }$, but

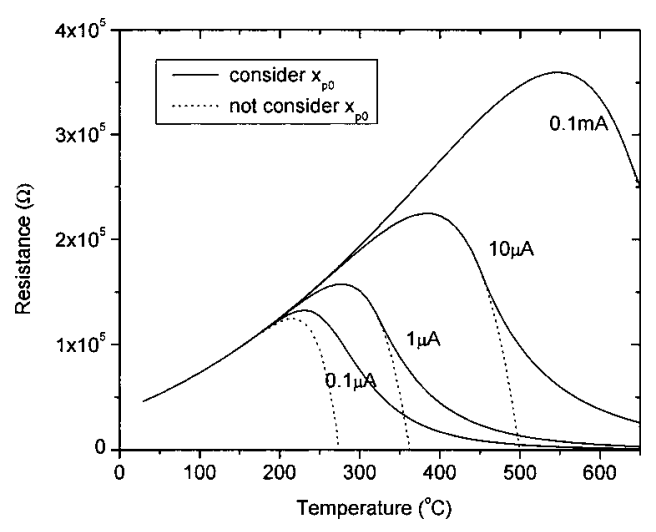

FIG. 8. Effects of equilibrium carrier concentrations on sensor resistance at bias currents of $0.1 \mu \mathrm{A}, 1 \mu \mathrm{A}, 10 \mu \mathrm{A}$, and $0.1 \mathrm{~mA}$. The $N_{D}$ is 1 $\times 10^{15} \mathrm{~cm}^{-3}$. Solid lines show the results with the equilibrium values in the carrier-concentration profile inside the sensor, whereas dotted lines are the results without the equilibrium concentrations.

different resistance values only at high temperatures beyond $T_{\max }$. This is because when the bias current is large, the carrier exclusion effect is strong and the hole concentration at high temperature is normally lower than the equilibrium level $p_{0}$ over most of the integration range from 0 to $L$. Therefore, $x_{\mathrm{p} 0}$ is normally larger than $L$ or very close to $L$, and hence there is no big difference between Eq. (12) and Eq. (17).

\section{E. Case 5}

With the inclusion of the effect of carrier concentrations on carrier mobilities, an iteration method is required to solve for $p$ in Eq. (6), $\mu_{\mathrm{n}}, \mu_{\mathrm{p}}$, and then $R$ in Eq. (17). In this calculation, the initial guesses of $b$ and $R$ are 2.8 and $100 \mathrm{k} \Omega$, respectively, and the maximum errors of $b$ and $R$ are both set at $1 \%$. Figure 9 shows that the carrier-concentration dependence of carrier mobilities has little effect on the sensor resistance for all bias currents. The reason is that when the temperature is lower than $T_{\max }$, the carrier exclusion effect dominates. The hole concentration is negligible and the electron concentration is nearly equal to the doping level over the entire $n$ region. This leads to the small effect of the

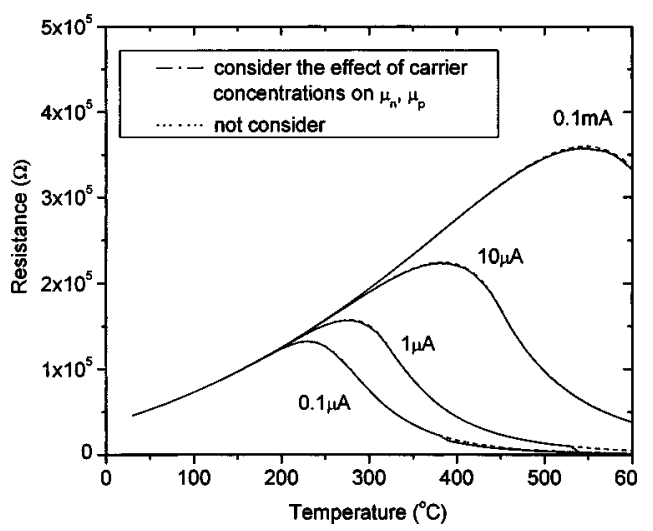

FIG. 9. Effects of carrier concentration-sensitive mobilities on sensor resistance at bias currents of $0.1 \mu \mathrm{A}, 1 \mu \mathrm{A}, 10 \mu \mathrm{A}$, and $0.1 \mathrm{~mA}$. The $N_{D}$ is 1 $\times 10^{15} \mathrm{~cm}^{-3}$. Solid lines are the results with carrier mobilities dependent on carrier concentrations, whereas dotted lines show the results without this dependence. 
carrier concentrations on the carrier mobilities. On the other hand, when the temperature is higher than $T_{\max }$, the effect of temperature overwhelms the effect of the carrier concentrations on the carrier mobilities, thus dominating the temperature dependence of the sensor resistance.

In summary, Eq. (12) is a simple and reasonably accurate formula for describing the sensor resistance for temperatures below $T_{\max }$, which covers the normal operating temperature range of the sensor.

\section{CONCLUSION}

An analytical device model is developed to explain the characteristics of the temperature sensor built on thin-film SOI. This model shows that at higher bias currents, the device has higher maximum operating temperature due to larger minority-carrier exclusion length. Compared with the results from the device simulator PISCES, this model is accurate for bias currents below $0.1 \mathrm{~mA}$. However, for larger bias currents, $T_{\max }$ calculated by the proposed model is larger than that simulated by PISCES due to the fact that highcurrent carrier injection at the $n^{+} n$ junction is neglected. When the bias current is low, a two-region model is proposed to improve the resistance model, and reasonable results are achieved. The carrier-mobility ratio and carrier-mobility dependence on carrier concentrations are found to have little effects on the device resistance for temperatures below $T_{\max }$. In summary, the device model presented here can be used to help understand the principle of the minority-carrier exclusion effect and guide the design of temperature sensors built on thin silicon film for high-temperature applications.

\section{ACKNOWLEDGMENTS}

This work is financially supported by the RGC grant of Hong Kong and the CRCG grant of the University of Hong Kong.

${ }^{1}$ J. L. Robert, S. Contreras, J. Camassel, J. Pernot, E. Weyret, and L. Dicioccio, Sens. Actuators, A 97-98, 27 (2002).

${ }^{2}$ E. Kohn, W. Ebert, M. Adamschik, P. Schmid, and A. Denisenko, New Diamond Front. Carbon Technol. 11(2), 81 (2001).

${ }^{3}$ J. L. Kennedy and N. Djeu, Sens. Actuators, A 100, 187 (2002).

${ }^{4}$ S. M. Sze, Physics of Semiconductor Devices, 2nd ed. (Wiley, New York, 1981), 27

${ }^{5}$ G. Raabe, NTG Fachberichte 79, 248 (1982).

${ }^{6}$ Silicon Temperature Sensors Databook (Philips, Eindhoven, 1990).

${ }^{7}$ S. R. in't Hout and S. Middelhoek, Sens. Actuators, A 60, 14 (1997).

${ }^{8}$ S. R. in't Hout and S. Middelhoek, Sens. Actuators, A 37, 38, 26 (1993).

${ }^{9}$ P. T. Lai and B. Y. Liu, Sens. Actuators, A 58, 85 (1997).

${ }^{10}$ P. T. Lai, B. Li, C. L. Chan, and J. K. O. Sin, IEEE Electron Device Lett. 20(11), 589 (1999).

${ }^{11}$ B. Li, P. T. Lai, and J. K. O. Sin, in Sensors 2002, Proceedings of IEEE, Vol. 2, 1419

${ }^{12}$ TMA, Medici Handbook (TMA, Palo Alto, 1993). 